\title{
Changes in soluble fibrin levels during the perioperative period of gynecologic cancer surgery
}

\author{
JUNICHI KODAMA, NORIKO SEKI, CHIKAKO FUKUSHIMA, TOMOYUKI KUSUMOTO, \\ KEIICHIRO NAKAMURA, ATSUSHI HONGO and YUJI HIRAMATSU
}

\begin{abstract}
Department of Obstetrics and Gynecology, Okayama University Graduate School of Medicine, Dentistry and Pharmaceutical Sciences, Okayama 700-8558, Japan
\end{abstract}

Received February 16, 2012; Accepted May 30, 2012

DOI: $10.3892 / \mathrm{ol} .2012 .891$

\begin{abstract}
The purpose of this study was to evaluate the changes in plasma soluble fibrin (SF) levels over time in gynecologic cancer patients following surgery. Furthermore, we examined the duration of the coagulation stage and determined a suitable duration for which thromboprophylaxis with anticoagulant agents should be administered. We retrospectively studied 311 patients with invasive gynecologic cancer who underwent surgery at Okayama University Hospital, Japan. The plasma SF levels were measured serially prior to the operation and on postoperative days $0,1,3,5,7,10,14,21$ and 28 . The plasma SF levels increased rapidly, peaked on postoperative day 1 and then decreased. The SF levels of patients with venous thromboembolism (VTE) were significantly different from those of VTE-negative patients on postoperative days 0-10. The SF levels on each day did not significantly differ between patients treated with chemical anticoagulants and those treated mechanically. The plasma SF levels were elevated $(\geq 7.0 \mu \mathrm{g} / \mathrm{ml})$ in 159 of the 311 patients $(51.1 \%)$ on one of the days when these levels were measured. Among the patients with elevated plasma SF levels, 110 patients $(69.2 \%)$ peaked on days $0-3$ and only 9 patients $(5.7 \%)$ peaked on days 21-28. Although only 1 of the 14 patients $(7.1 \%)$ who showed peak levels on day 14 had undergone chemotherapy following surgery, 8 of the 9 patients (88.9\%) whose levels peaked on days 21-28 had undergone chemotherapy following surgery $(\mathrm{P}=0.0002)$. In conclusion, the plasma SF levels increased rapidly, peaked on postoperative day 1 and then decreased. These levels peaked within 14 days of surgery in most cases. Therefore, chemical thromboprophylaxis may be administered for at least up to 14 days following surgery.
\end{abstract}

Correspondence to: Dr Junichi Kodama, Department of Obstetrics and Gynecology, Okayama University Graduate School of Medicine, Dentistry and Pharmaceutical Sciences, 2-5-1 Shikata-cho, Kita-ku, Okayama 700-8558, Japan

E-mail: kodama@cc.okayama-u.ac.jp

Key words: soluble fibrin, venous thromboembolism, thromboprophylaxis, gynecologic cancer

\section{Introduction}

Cancer is widely accepted as a risk factor for venous thromboembolism (VTE) and, to a lesser extent, arterial thrombosis. This risk is attributable to factors such as the expression of prothrombotic factors and compression of the blood vessels by tumors, inflammatory response to malignancies by the host, immobility, surgery, indwelling central venous catheters, and certain antitumor therapies (1). In surgical oncology patients, VTE is the predominant cause of mortality during the first 30 postoperative days (2). VTE is also a common complication associated with gynecologic cancer surgery, and patients with gynecological malignancies are classified under the highestrisk group (3).

Coagulation and fibrinolysis in the blood are complex processes, and numerous markers of thrombin and plasmin action in patients with VTE have been identified (4). Soluble fibrin (SF) and D-dimer are sensitive markers for VTE. D-dimer is a stable end-product of fibrin degradation, and D-dimer levels increase as a result of fibrin formation and fibrinolysis. Conversely, SF reflects thrombi activation and the cleavage of fibrinogen during the early stage of disease and is used as an indicator of coagulation (5). We recently reported the changes in plasma D-dimer levels over time in gynecologic cancer patients following surgery (6); however, changes in the SF levels in these patients following surgery have not been elucidated.

The purpose of the present study was to evaluate the changes in the plasma SF levels over time in gynecologic cancer patients following surgery. Furthermore, we examined the duration of the coagulation stage and determined a suitable duration for which chemoprophylaxis with anticoagulant agents should be administered.

\section{Materials and methods}

Study population. We studied 311 patients with invasive gynecologic cancer who had undergone surgery at Okayama University Hospital between August 2007 and August 2011. The study was approved by the ethics committee of Okayama University Graduate School of Medicine, Okayama, Japan. Informed consent was obtained from each patient prior to blood collection. The median age of the patients was 55 years (range, 16-81 years). Patients with various types of gynecologic 


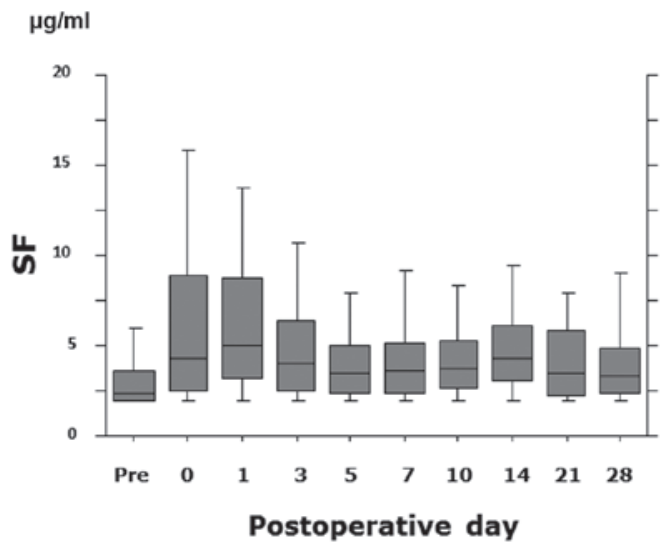

Figure 1. Course of plasma soluble fibrin (SF) levels in patients with gynecologic cancer $(n=311)$. The median is indicated within the boxplot by a horizontal line inside the box. The lower edge of the whisker, the lower end of the box, the upper end of the box, and the upper edge of the whisker represent the 10th, 25th, 75th and 90th percentiles, respectively.

cancers were enrolled: 65 patients with ovarian cancer, 142 with endometrial cancer, 103 with cervical cancer, and 1 with vulvar cancer. Patients with preoperative VTE were excluded. All the patients received general anesthesia. Calf-length external sequential compression devices (SCD) were placed on both legs of all the patients from the beginning of the surgery until after the operation. Chemical anticoagulants were administered to 215 patients following surgery: 54 patients were treated with unfractionated heparin (UFH; 10,000 U/day); 50 patients with fondaparinux (FPX; $2.5 \mathrm{mg} /$ day); and 111 patients with enoxaparin (ENO; 4,000 U/day). UFH, ENO and FPX were administered for a median of 5 days (range, 3-23 days), 10 days (range, 1-15 days), and 10 days (range, 3-20 days), respectively. UFH was used at the discretion of the surgeon before regulatory approval of the other agents was obtained (i.e., before 2008), and ENO and FPX were routinely used after 2009. In the present study population, VTE was detected in 23 patients $(7.4 \%)$.

Measurement of plasma SF levels. The plasma SF levels were measured serially prior to surgery and on postoperative days 0 , $1,3,5,7,10,14$ and 21; these levels were also measured after day 28 . We used the latex agglutination method to measure the SF levels with IATRO SF (Mitsubishi Kagaku Iatron, Inc., Tokyo, Japan), which contains the monoclonal antibody IF-43 as the reagent. IF-43 recognizes a segment of the fibrin A $\alpha$ chain (A $\alpha-17-78$ residue segment) exposed in the $E$ region of the fibrin monomer (FM) when this FM binds to the D region of another FM or fibrinogen. The antibody is coated for the SF assay (7). The normal range is $<7.0 \mu \mathrm{g} / \mathrm{ml}$.

Statistical analyses. We used Fisher's exact test or the Mann-Whitney U-test to statistically analyze the differences between the groups. Probability values of $<0.05$ were considered to indicate statistical significance.

\section{Results}

Time course of postoperative plasma SF levels. The plasma SF levels increased rapidly, peaked on postoperative day 1
A

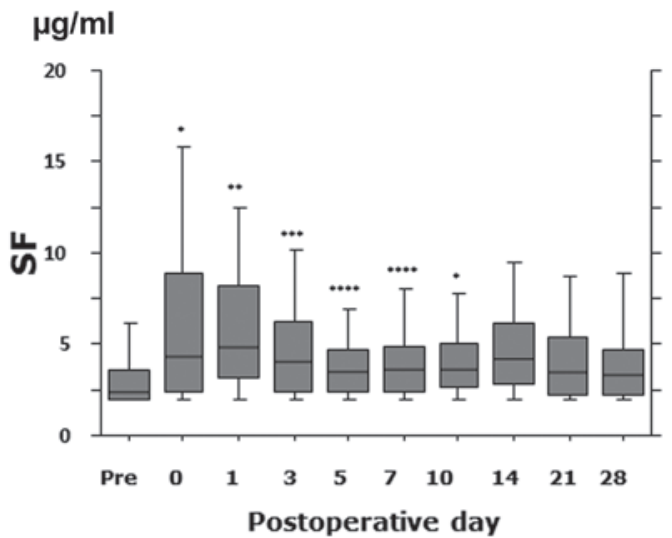

B

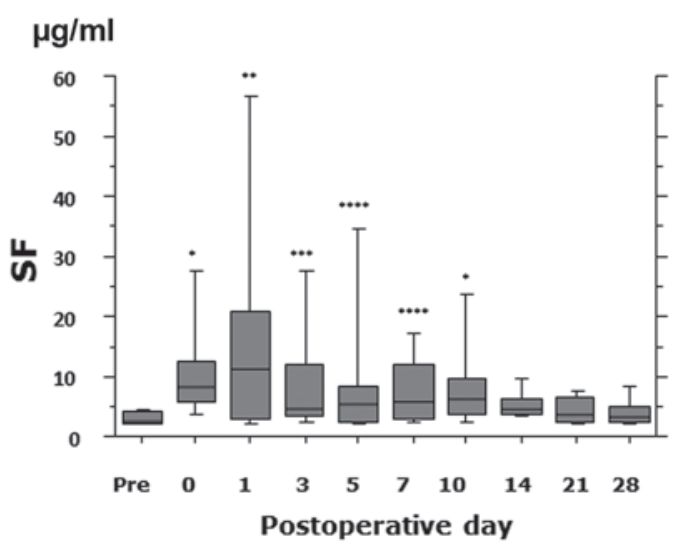

Figure 2. (A) Course of plasma soluble fibrin (SF) levels in patients without VTE $(n=288)$. (B) Course of plasma SF levels in patients with VTE $(n=23)$. ${ }^{*} \mathrm{P}<0.0001,{ }^{* *} \mathrm{P}=0.002,{ }^{* * * *} \mathrm{P}=0.02,{ }^{* * * *} \mathrm{P}=0.003$. VTE, venous thromboembolism.

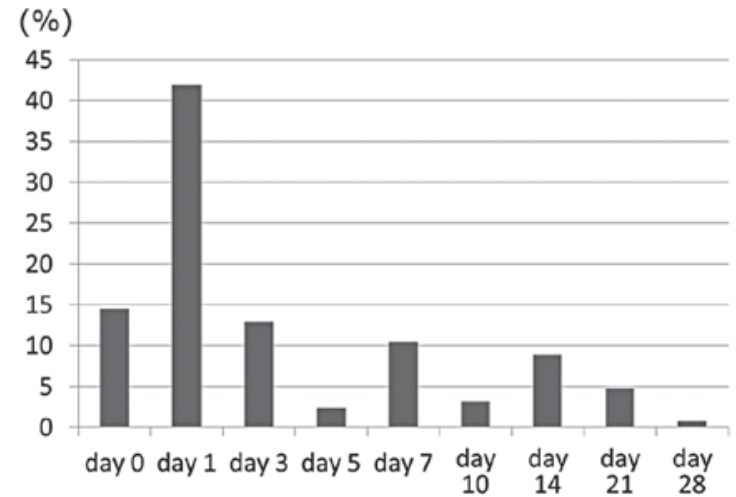

Figure 3. The peak day of plasma soluble fibrin (SF) levels in patients with gynecologic cancer $(\mathrm{n}=159)$.

(median, $4.6 \mu \mathrm{g} / \mathrm{ml}$ ), and then decreased (Fig. 1). The SF levels of VTE-positive patients were significantly different from those of VTE-negative patients on postoperative day 0 (medians $8.5 \mu \mathrm{g} / \mathrm{ml}$ and $4.3 \mu \mathrm{g} / \mathrm{ml}$, respectively; $\mathrm{P}<0.0001$ ), day 1 (medians $11.3 \mu \mathrm{g} / \mathrm{ml}$ and $4.9 \mu \mathrm{g} / \mathrm{ml}$, respectively; $\mathrm{P}=0.002$ ), day 3 (medians $4.4 \mu \mathrm{g} / \mathrm{ml}$ and $4.0 \mu \mathrm{g} / \mathrm{ml}$, respectively; $\mathrm{P}=0.02$ ), day 5 (medians $5.6 \mu \mathrm{g} / \mathrm{ml}$ and $3.5 \mu \mathrm{g} / \mathrm{ml}$, respectively; $\mathrm{P}=0.003$ ), day 7 (medians $5.8 \mu \mathrm{g} / \mathrm{ml}$ and $3.6 \mu \mathrm{g} / \mathrm{ml}$, respectively; $\mathrm{P}=0.003$ ), and day 10 (medians $6.1 \mu \mathrm{g} / \mathrm{ml}$ and $3.6 \mu \mathrm{g} / \mathrm{ml}$, respectively; $\mathrm{P}<0.0001$ ) (Fig. 2). The SF levels on each day did not significantly differ between the 
patients treated with chemical anticoagulants and those treated mechanically (data not shown).

Incidence and peak day of elevated plasma SF. The plasma SF levels were elevated $(\geq 7.0 \mu \mathrm{g} / \mathrm{ml})$ in 159 of the 311 patients $(51.1 \%)$ on one of the days when the levels were measured. The days on which the plasma SF levels peaked are shown in Fig. 3. The plasma SF levels of 67 patients $(42.1 \%)$ peaked on day 1,23 patients $(14.5 \%)$ on day 0 , and 20 patients $(12.6 \%)$ on day 3 . However, in 14 patients $(8.8 \%)$, these levels peaked on day 14 and in 9 patients $(5.7 \%)$ on days $21-28$. We observed that only 1 of the 14 patients $(7.1 \%)$ whose levels peaked on day 14 had undergone chemotherapy following surgery. However, chemotherapy had been administered to 8 of the $9(88.9 \%)$ patients whose levels peaked on days 21-28 ( $\mathrm{P}=0.0002)$.

\section{Discussion}

The SF levels reflect the changes that occur during the early phase of a thrombotic event, while D-dimer levels reflect secondary fibrinolysis after clot formation (8). Elevated levels of circulating plasma SF indicate the conversion of fibrinogen to fibrin by thrombin. Plasma SF levels rapidly increase and then decrease relatively soon following orthopedic surgery $(9,10)$. In a previous study, we reported that plasma D-dimer levels gradually increased, peaked on postoperative days 7-10, and then decreased (6). To the best of our knowledge, changes in the plasma SF levels in gynecologic cancer patients have not yet been described. In the present study, we measured the plasma SF levels longitudinally and demonstrated that these levels increased rapidly following surgery, peaked on postoperative day 1, and then decreased. This observation suggests that increased intravascular fibrin formation had already occurred during perisurgery. High plasma levels of D-dimer are maintained for longer periods than those of SF because the half-life of plasma D-dimer is relatively long. Furthermore, we demonstrated that the SF levels in the VTE-positive patients were significantly higher than those in the VTE-negative patients on postoperative days 0-10. Therefore, hypercoagulation may exist from surgery to at least 10 days after surgery in certain cases. It is reported that the plasma concentration of SF following orthopedic surgery is significantly higher in patients with VTE than in those without VTE on days 1, 4 and 14 (10).

In approximately $70 \%$ of the patients, the plasma SF levels peaked within 3 days of surgery. Kearon reported that in a large number of cases, VTE occurred between the intraoperative period and postoperative day 3 (11). The findings of the present study support this finding. In approximately $95 \%$ of the patients, the plasma SF levels peaked within 14 days of surgery. This finding suggests that the risk of symptomatic VTE is generally highest during the 2 weeks following surgery, and therefore, prophylaxis should be administered to patients with gynecologic cancer for at least 2 weeks postoperatively.

In the ENOXACAN II study, a double-blind, multicenter clinical trial involving patients undergoing open abdominal or pelvic cancer surgery, the incidence of VTE decreased significantly from 12 to $4.8 \%$ when inpatient thromboprophylaxis was continued for 27-31 days (12). Most guidelines recommend that cancer patients undergoing elective major abdominal or pelvic surgery should receive prophylaxis in the hospital and after discharge for up to 1 month after surgery (13). However, the exact indications for such extended prophylaxis have not been defined. In fact, findings of a systematic review showed that extended prophylaxis reduced asymptomatic VTE without decreasing the mortality rate at 3 months, and that the evidence for extended regimens was limited and of poor quality (13). Notably, approximately $90 \%$ of the patients whose plasma SF levels peaked on days 21-28 had undergone chemotherapy following surgery. Therefore, the elevated plasma SF levels were probably not due to surgery, but instead associated with chemotherapy. Peedicayil et al reported that $75 \%$ of VTE events occur more than a week after surgery and $36 \%$ occur after 4 weeks (14). They believed that early VTE was due to the surgery, while late VTE reflected changes during the period of recovery and initiation of adjuvant treatments such as chemotherapy.

Our current findings proved that the plasma SF levels increased rapidly, peaked on postoperative day 1 , and then decreased. Furthermore, we showed that the levels peaked before postoperative day 14 in most cases. Therefore, postoperative chemical thromboprophylaxis may be administered for at least up to 14 days after surgery. Further study is required to determine which patients should be considered for extended prophylaxis.

\section{References}

1. Lin A, Ryu J, Harvey D, et al: Low-dose warfarin does not decrease the rate of thrombosis in patients with cervix and vulvo-vaginal cancer treated with chemotherapy, radiation, and erythropoietin. Gynecol Oncol 102: 98-102, 2006.

2. Bradley CT, Brasel KJ, Miller JJ, et al: Cost-effectiveness of prolonged thromboprophylaxis after cancer surgery. Ann Surg Oncol 17: 31-39, 2010.

3. Einstein MH, Pritts EA and Hartenbach EM: Venous thromboembolism prevention in gynecologic cancer surgery: a sysytematic review. Gynecol Oncol 105: 813-819, 2007.

4. Suzuki A, Ebinuma H, Matsuo M, et al: The monoclonal antibody that recognizes an epitope in the C-terminal region of the fibrinogen alpha-chain reacts with soluble fibrin and fibrin monomer generated by thrombin but no with those formed as plasmin degradation products. Thromb Res 121: 377-385, 2007.

5. Tsuji A, Wada H, Matsumoto T, et al: Elevated levels of soluble fibrin in patients with venous thromboembolism. Int J Haematol 88: 448-453, 2008.

6. Kodama J, Seki N, Masahiro S, et al: D-dimer level as a risk factor for postoperative venous thromboembolism in Japanese women with gynecologic cancer. Ann Oncol 21: 1651-1656, 2010.

7. Soe G, Kohno I, Inuzuka K, et al: A monoclonal antibody that recognizes a neo-antigen exposed in the $E$ domain of fibrin monomer complexed with fibrinogen or its derivatives: its application to the measurement of soluble fibrin in plasma. Blood; 88 : 2109-2117, 1996.

8. Bounameaux H, Cirafici P, de Moerloose P, et al: Measurement of D-dimer in plasma diagnostic aid in suspected pulmonary embolism. Lancet 337: 196-200, 1991.

9. Misaki T, Kitajima I, Kabata T, et al: Changes of the soluble fibrin monomer complex level during the perioperative period of hip replacement surgery. J Orthop Sci 13: 419-424, 2008.

10. Sudo A, Wada H, Nobori T, et al: Cut-off values of D-dimer and soluble fibrin for prediction of deep vein thrombosis after orthopaedic surgery. Int J Hematol 89: 572-576, 2009.

11. Kearon C: Duration of venous thromboembolism prophylaxis after surgery. Chest 124: 386S-392S, 2003.

12. Bergqvist D, Agnelli G, Cohen AT, et al: Duration of prophylaxis against venous thromboembolism with enoxaparin after surgery for cancer. N Engl J Meed 346: 975-980, 2003.

13. Akl EA, Terrenato I, Barba M, et al: Extended perioperative thromboprophylaxis in patients with cancer. Thromb Haemost 100: 1176-1180, 2008.

14. Peedicayil A, Weaver A, Li X, et al: Incidence and timing of venous thromboembolism after surgery for gynecological cancer. Gynecol Oncol 121: 64-69, 2011. 